\section{CANCER}

\section{Enhancing EGFR targeting}

The epidermal growth factor receptor (EGFR) is altered in $50 \%$ of glioblastomas (GBMs), but EGFR inhibitors have yielded disappointing results in clinical assays. A new study may allow improved targeting of EGFR-dependent GBMs by identifying a key regulator of EGFR in GBMs (J. Clin. Invest. http://dx.doi.org/ 10.1172/JCI63623).

Natividad Pozo et al. examined the effect of DYRK1A, a kinase important in central nervous system development, on EGFR in established GBM cell lines. They found that silencing of DYRK1A led to a reduction in the amounts of EGFR and inhibited self-renewal of the cells. Downregulation of DYRK1A also decreased tumor growth in nude mice after injection of GBM cells. Similarly, the authors found that pharmacological inhibition of DYRK $1 A$ reduced self-renewal of GBM cells and decreased tumor growth in nude mice injected with GBM cells.

The researchers then turned their attention to human glioma samples, finding that DYRK1A was more highly expressed in gliomas compared with normal brain tissue. They also identified a positive correlation between EGFR and DYRK1A expression in GBM samples.

Notably, the authors' data indicate that DYRK1A acts at the level of regulating EGFR stability rather than its kinase activity. They suggest that targeting DYRK1A, either alone or in combination with EGFR inhibitors, could have therapeutic benefit in EGFR-dependent GBMs. - MS

\section{NEURODEVELOPMENTAL DISORDERS}

\section{A therapy for Pretzel syndrome}

Treatment with the immunosuppressive drug rapamycin may have a therapeutic effect in patients with Pretzel syndrome, a rare neurodevelopmental disorder (Sci. Trans. Med. 5, 182ra53).

Pretzel syndrome is characterized by epilepsy, cognitive delay and a series of neuroanatomical abnormalities. The disease is caused by mutations in $\angle Y K 5$, which encodes an inhibitor of the mammalian target of rapamycin complex 1 (mTORC1). In mice, loss of Lyk5 affects neuronal migration and leads to the formation of clusters of misplaced neurons in the cortex similar to those observed in patients with Pretzel syndrome.

Whitney Parker et al. found that mTORC1 inhibition with rapamycin can prevent the

\title{
AUTISM SPECTRUM DISORDERS
}

\section{Mutual mutation mechanisms}

Researchers have identified altered endocannabinoid signaling as a common mechanism by which different mutations in the gene that encodes the synaptic protein neuroligin- 3 could cause autism (Neuron 78, 498-509).

Several mutations in the gene that encodes neuroligin-3 have been linked to autism, including a deletion mutation and a substitution at amino acid 451

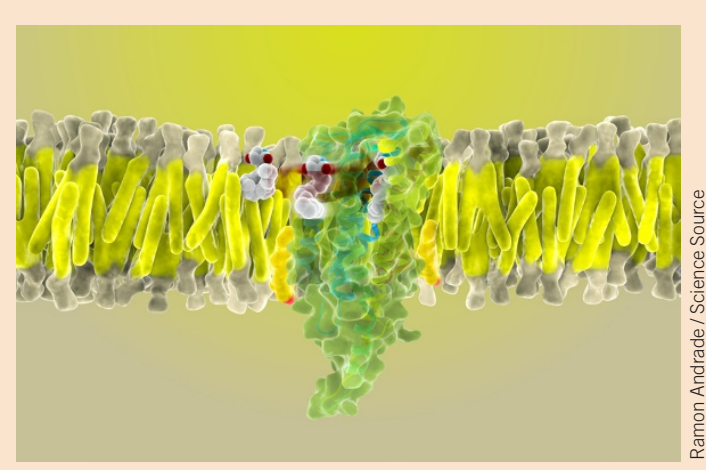
of the protein. Csaba Földy et al. examined the electrophysiological properties of synapses from inhibitory interneurons onto pyramidal neurons in the hippocampus with the goal of identifying synaptic dysfunction that would be commonly induced by both the mutations, possibly leading to autism. The researchers found that inhibitory neurotransmission from cholecystokinin-positive interneurons was enhanced in brain slices from mice with either mutation, whereas this was not the case with parvalbumin-positive interneurons.

Cholecystokinin interneurons express cannabinoid receptors, and deficits in cannabinoid signaling can enhance inhibitory neurotransmission. Földy et al. found that a cannabinoid receptor antagonist could enhance inhibitory neurotransmission in brain slices from wild-type mice, but could not do so in brain slices from either of the neuroligin-3-mutant mice, suggesting that they both had deficits in endocannabinoid signaling. How this mechanism could lead to the behavioral phenotypes associated with autism remains to be explored. $-E C$

cortical malformations caused by Lyk5 depletion in the mouse. As rapamycin also prevented a migration defect found in fibroblasts from patients with Pretzel syndrome, the authors treated five patients with rapamycin and reported that this resulted in a reduction in seizure frequency.

Although these findings need to be replicated in a larger cohort in a blinded trial, the results raise the possibility that $\mathrm{mTORC} 1$ inhibition may be useful for treating patients with this neurodevelopmental condition - JCL

\section{CARDIOVASCULAR DISEASES}

\section{Young blood helps the heart}

Enlargement of the heart-cardiac hypertrophy-occurs with aging and is thought to contribute to diastolic heart failure, a common form of heart failure in the elderly. Richard Lee and his colleagues now identify the cytokine GDF11 as a circulating antihypertrophic factor in mice whose levels decrease with age (Cell 153, 828-839).

In parabiosis experiments, in which the blood circulations of an old mouse and a young mouse are connected, the researchers found that cardiac hypertrophy in the old mouse was reversed after 4 weeks. The authors found a number of proteins whose abundance differed between blood plasma from young versus old mice. One of these was GDF11, which showed widespread tissue expression, with the highest levels in the spleen, and whose levels in both blood and spleen were higher in young mice as compared to old mice The researchers carried out functional studies to show that GDF11 inhibits cardiac hypertrophy of cultured cardiomyocytes and reverses cardiac hypertrophy in old mice. Raising the levels of GDF11 in the blood might therefore be beneficial for the aged heart. $-M B$

AGING

\section{A new target in progeria}

A recent study suggests that targeting the enzyme isoprenylcysteine carboxyl methyltransferase (ICMT) might have therapeutic benefit in a mouse model of a premature aging disease (Science http://dx.doi.org/10.1126/ science.1238880).

A number of progeroid disorders are caused by the accumulation of an aberrant form of prelamin $A$ at the nuclear envelope, leading to misshapen nuclei. Prelamin A is post translationally modified at its $C$ terminal by farnesylation and subsequent ICMT-mediated 
methylation. Mohamed X. Ibrahim et al. investigated the role of ICMT in the disease phenotype of a mouse model of progeria caused by knockout of the gene Zmpste24. Taking advantage of a hypomorphic allele of $/ \mathrm{cmt}\left(/ \mathrm{cm} \mathrm{t}^{\mathrm{hm}}\right)$, they found that Zmpste24-/-; $/ \mathrm{cm} \mathrm{t}^{\mathrm{hm} / \mathrm{hm}}$ mice showed improvement in several symptoms associated with progeria compared with Zmpste24-/- mice that had normal expression of $1 \mathrm{cmt}$.

In fibroblasts from the Zmpste $24^{-1-} ; \mathrm{lcm} \mathrm{thm}^{\mathrm{hm}}$ mice, prelamin A was relocalized away from the nuclear rim, although the nuclei were still misshapen. In contrast to Zmpste24-/- fibroblasts, which proliferate slowly and enter senescence prematurely, Zmpste $24^{-/-} ;$Icmt $\mathrm{tm}^{\mathrm{hm}} \mathrm{hm}$ fibroblasts showed wild-type rates of proliferation. Mechanistically, these effects seem to be mediated by upregulation of AKT1-mTOR signaling in conditions of $/ \mathrm{cm}$ t deficiency. The authors then extended these findings to the human situation by inhibiting ICMT in cells from people with Hutchinson-Gilford progeria syndrome (HGPS) using either shRNAs or a pharmacological inhibitor, showing that, as in the mouse cells, ICMT inhibition resulted in increased proliferation and delayed senescence. $-M S$

\section{MALARIA}

\section{Malaria network using vesicles}

Plasmodium, the parasite responsible for malaria, must differentiate once in its life cycle to the sexual stage form, called the gametocyte, to ensure transmission to other hosts. Two new studies show that malariainfected red blood cells communicate with each other by vesicles, which induces gametocyte production and host immunomodulation (Ce// http://dx.doi.org/10.1016/ j.cell.2013.04.029; Cell Host Microbe 13, 521-534, 2013).

The two groups provided insights into how blood-stage Plasmodium falciparum communicates in the host. Neta Regev-Rudzki et al. showed that the release of exosome-like vesicles from infected red blood cells before parasite egress enables transfer of genes between parasites. This not only allows parasites to exchange drug resistance genes to promote their survival but also signaling that allows the parasites to differentiate into their sexual forms. Pierre-Yves Mantel et al. found that a larger type of vesicle, microvesicles, caused an increase in gametocyte numbers and also activated innate immune cells to release proinflammatory cytokines, which might contribute to disease. These vesicles contained red blood cell-derived proteins and parasite antigens, but it is unknown what other cargo may be inside and what proteins are responsible for triggering differentiation into the sexual stage.

The studies also show that $P$. falciparuminfected red blood cells can internalize exosomes and microvesicles and that this uptake increases in conditions of stress and when the blood concentration of vesicles increases. This suggests that parasite crosstalk may lead to optimized gametocyte production and transmission to mosquitoes. This vesiclemediated transport could be targeted to block parasite transmission and reduce disease progression. $-C P$

\section{IMMUNOLOGY \\ Restraining natural killer cells}

Regulatory $T$ cells ( $T_{\text {reg }}$ cells) suppress the activity of several immune cell types; however, the direct mechanism whereby they control natural killer (NK) cell function remains unclear. Three studies suggest $T_{\text {reg }}$ cells may attenuate NK cell activity by limiting the amount of interleukin-2 (IL-2) available.

In a mouse model of type 1 diabetes, Jonathan Sitrin et al. (J. Exp. Med. http://dx.doi.org/10.1084/jem.20122248) show that ablation of $T_{\text {reg }}$ cells induces the accumulation and activation of NK cells in the pancreas, leading to diabetes. Inhibition of IL-2, a cytokine previously shown to promote NK cell proliferation and interferon- $\gamma$ (IFN- $\gamma$ ) production, reduced NK cell infiltration into the pancreas and their production of IFN- $\gamma$.

Georg Gasteiger et al. (J. Exp. Med. http://dx.doi.org/10.1084/jem.20122462; J. Exp. Med. http://dx.doi.org/10.1084/ jem.20122571) report that $\mathrm{T}_{\text {reg }}$ cell depletion induces systemic autoimmunity, leading to increased NK cell cytotoxicity toward missing self targets. This activity was dependent on IL-2, as inhibition of IL-2 or depletion of $\mathrm{CD}^{+}$cells, a dominant source of IL-2, reduced NK cell activity. Ablation of $T_{\text {reg }}$ cells also resulted in IL-2-dependent expansion of an immature subset of CD127+ NK cells. Taken together, these findings highlight how adaptive immune responses can control innate immune cell homeostasis and activation in the steady state and in disease. $-K D S$

Written by Michael Basson, Eva Chmielnicki,

Kevin Da Silva, Juan Carlos López, Carolina Pola and Meera Swami

\section{New from NPG}

Hypothalamic programming of systemic ageing involving IKK- $\beta$, NF-кB and GnRH Zhang, G. et al. Nature http://dx.doi. org/10.1038/nature12143 (1 May)

The authors show that aging in mice is regulated by hypothalamic nuclear factor- $\kappa \mathrm{B}(\mathrm{NF}-\kappa \mathrm{B})$ signaling. This pathway leads to decreased amounts of gonadotropin-releasing hormone (GnRH) and reduced neurogenesis. Administration of $\mathrm{GnRH}$ stimulated neurogenesis in adult mice and slowed aging.

The collagen receptor discoidin domain receptor 2 stabilizes SNAIL1 to facilitate breast cancer metastasis

Zhang, K. et al. Nat. Cell Biol. http://dx.doi. org/10.1038/ncb2743 (5 May)

This study shows that stabilization of SNAIL1 by discoidin domain receptor 2 (DDR2) promotes breast cancer metastasis. DDR2 expression associated with nuclear SNAIL1 was found in a number of human invasive ductal breast carcinomas.

Multiple populations of artemisinin-resistant Plasmodium falciparum in Cambodia Miotto, O. et al. Nat. Genet. http://dx.doi. org/10.1038/ng.2624 (28 April)

By sequencing whole genomes of P. falciparum isolated from 825 malaria cases, the authors were able to characterize patterns of genetic variation and drug resistance, as well as genetic markers that can identify resistant strains.

T cells maintain an exhausted phenotype after antigen withdrawal and population reexpansion

Utzchneider, D.T. et al. Nat. Immunol. http:// dx.doi.org/10.1038/ni.2606 (5 May)

The authors find that 'exhausted' CD8+ $T$ cells transferred from chronically lymphochoriomeningitis virus (LCMV)infected mice to naive mice could proliferate and control a viral infection. The progeny of the transferred cells still expressed exhaustion markers in the absence of chronic infection, suggesting that the exhausted phenotype may represent a stable differentiation state.

GABA progenitors grafted into the adult epileptic brain control seizures and abnormal behavior

Hunt, R.F. et al. Nat. Neurosci. http://dx. doi.org/10.1038/ni.3392 (5 May)

Grafting the precursors of GABAproducing inhibitory neurons into the hippocampus of adult epileptic mice reduced seizures and ameliorated behavioral deficits. 